\title{
Polarity Effects on Breakdown of Short Gaps in a Point- plane Topology in Air
}

\author{
M. G. Hogg, I. V. Timoshkin, S. J. McGregor, M. P. Wilson and M. J. Given \\ Department of Electronic and Electrical Engineering, University of Strathclyde \\ 204 George Street, Glasgow, G1 1XW, UK
}

\begin{abstract}
Electrical breakdown in air in a point-plane topology involves complex processes that are still not fully understood. Unlike uniform-field topologies, the highly-divergent fields produced by point-plane topologies create pre-breakdown corona with volumetric space charge. It is known that space charges developed by corona discharge have significant impacts on the breakdown voltage in non-uniform electrode topologies. With large inter-electrode gaps $(>\mathrm{cm})$ the breakdown voltage for a $\mathrm{HV}$ point cathode in air at atmospheric pressure is noticeably larger than a HV point anode. However, this paper shows that in shorter point-plane gaps in air (less than $\sim 10 \mathbf{~ m m}$ ), in the air pressure range 0.1-0.35 MPa, an $\mathrm{HV}$ point anode has a similar breakdown voltage which eventually is surpassed by the $\mathrm{HV}$ point cathode as the inter-electrode gap is increased. The inter-electrode gap at which the HV cathode has a higher hold-off voltage is found to be dependent on the gas pressure and radius of the point electrode.
\end{abstract}

Index Terms - Electrical breakdown, Corona discharge, Air-filled plasma-closing switch, Point-plane electrode topology.

\section{INTRODUCTION}

WITHIN the power and pulsed power industries there is a need for high voltage (HV) systems with a reduced footprint and, as such, it is useful to design and characterise compact gas-filled switches with short inter-electrode gaps. This paper investigates the effect of energisation polarity on the performance of a self-breakdown air-filled plasma closing switch with a point-plane electrode topology which is stressed with ramped voltage. Although electrical breakdown in air in point-plane electrode topologies has been under investigation for decades, further studies of the breakdown mechanisms and characteristics are required, as these topologies are widely used in practical applications in the power and pulsed power industries, [2-7]. In some cases, corona discharges are used for optimisation of switch operation characteristics and the present work investigates the effect of such pre-breakdown corona discharges of both polarities on the operation and performance of the air-filled plasma closing switch.

When dealing with highly non-uniform electric fields, corona discharges can be initiated before complete (spark) breakdown in the gap $[1,3,4,6,7,10]$. Corona discharge is a self-sustained discharge, initiated in high-field regions if

Manuscript received on 22 October 2014, in final form 16 April 2015, accepted 15 May 2015. the field exceeds the threshold corona ignition value $(\sim 30 \mathrm{kV} / \mathrm{cm}$ for a spherically capped rod or pin $\mathrm{HV}$ electrode in atmospheric air, [1]). The field magnitude reduces away from the sharp HV electrode, thus the ionisation zone of the corona discharge is defined by the critical ionisation field value, which is $\sim 25-30 \mathrm{kV} / \mathrm{cm}$ for atmospheric air [1], ionisations stops as soon as the field reduces below this critical value.

Corona discharges can be classified according to the polarity of voltage which stresses the sharp electrode. Negative corona discharges in air initiate and sustain in accordance with the Townsend discharge mechanism, with primary electron avalanches emanating from the tip of the cathode into the ionisation zone. Much slower positive ions travelling in the opposite direction develop a cathode sheath which increases the field in close proximity to the sharp cathode. In the case of corona discharges in electronegative gases, negative ions are produced by attachment of electrons to electronegative molecules (molecules of oxygen in the case of air), these ions travel towards the anode through the transport zone where the electric field is lower than the critical ionisation field. These ions produce the negative volumetric space charge which results in the reduction of the space charge saturated electric field in the 
vicinity of the $\mathrm{HV}$ electrode as compared with the Laplacian electric field.

Positive corona initiates as a number of smaller-scale electron avalanches directed towards the sharp anode, and the secondary photoemission of electrons plays an important role in the development of this type of corona discharge. The electrons are absorbed into the anode leaving behind positive ions resulting in development of the positive local charge in close proximity to the anode and a reduction of the field at the anode. Thus, the ignition voltage of positive corona discharges in atmospheric air is higher than the ignition voltage of negative ones, [10].

Positive ions travel to the cathode through the transport zone providing a current in the discharge circuit, however, the volumetric positive space charge does not modify the field as noticeably as in the case of the negative corona in an electronegative gas: the positive corona current and therefore the density of positive ions is significantly lower than the negative corona current for the same applied voltage.

With further increase in the applied voltage, a spark breakdown can be developed in the inter-electrode gap, resulting in gap closure. In non-uniform topologies in electronegative gases (including air), it is common to expect a higher breakdown voltage for a negativelyenergised sharp HV electrode as compared with a positively-energised electrode, [11]. This is due to the development of negative volumetric space charge (as discussed above), which results in field screening in the vicinity of the sharp cathode, $[1,9]$. The field in the interelectrode gap is highly non-uniform: it reduces sharply from its maximum value near the cathode to a minimum value, then the field increases slightly toward the opposite plane electrode. For the point-pane electrode topology stressed with DC voltage in atmospheric air, the minimum value of the field is observed at distances $<1 \mathrm{~mm}$ away from the cathode, depending on the corona current, [12]. In the case of positive corona discharge, positive ions also modify the field distribution in the vicinity of the corona generating electrode (anode), however the degree of distortion of the field in the main gap is considered to be not so significant, [13].

Thus, for the longer inter-electrode distances which are sufficient for the development of noticeable space charges in the main inter-electrode space, the spark breakdown voltage in the case of negatively stressed high tension electrode can be higher than the breakdown voltage for a corresponding positively stressed electrode, [9, 14]. Therefore, higher DC or ramped switching voltages can be achieved using plasma closing switches with corona electrodes filled with electronegative gases, which is of significant importance to the pulsed power industry, $[1,8$, $9,18,19]$.

However, over shorter inter-electrode distances, the complex processes in the vicinity of the high-tension electrode including development of plasma in the ionization zone, sheath formation, field screening and the space charges can result in less pronounced difference (or

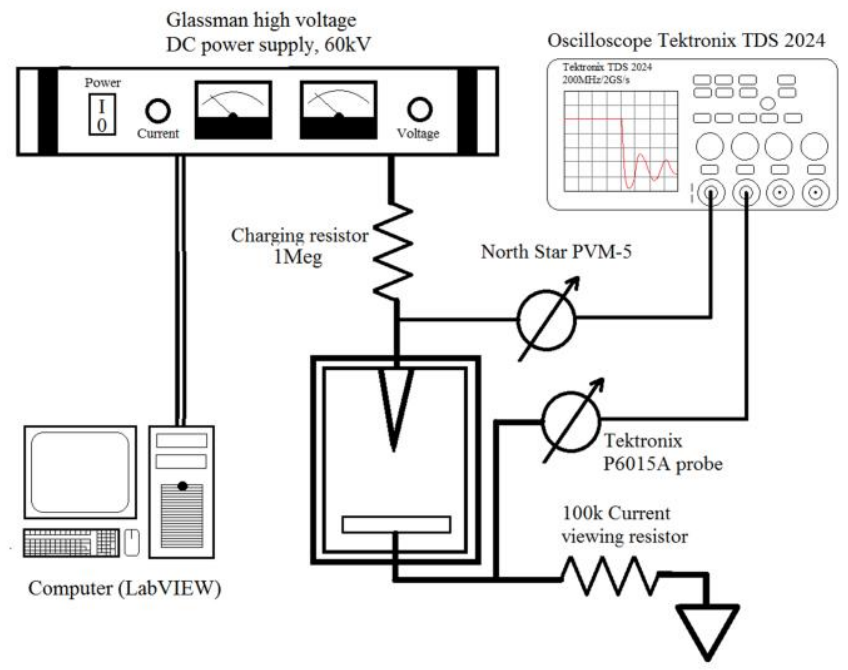

Figure 1. Experimental set up for measuring pre-breakdown corona current. Spark breakdown voltage was measured in the absence of $100 \mathrm{k} \Omega$ current viewing resistor.

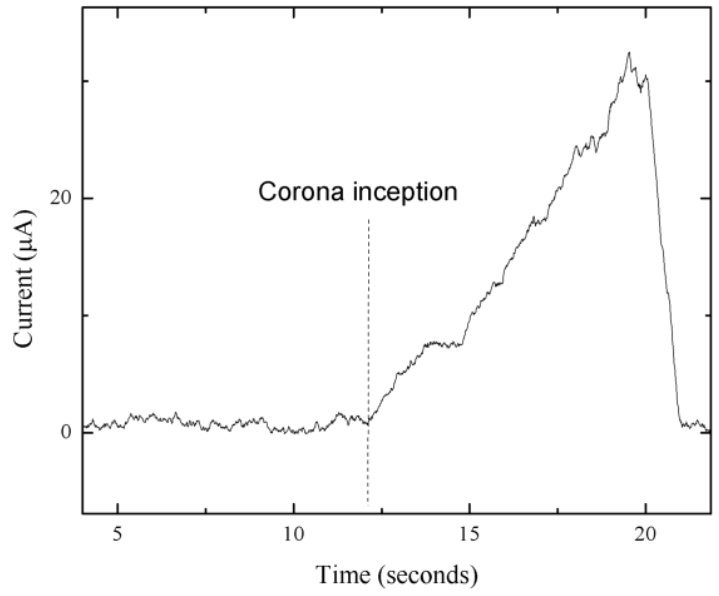

(a) Positive energisation

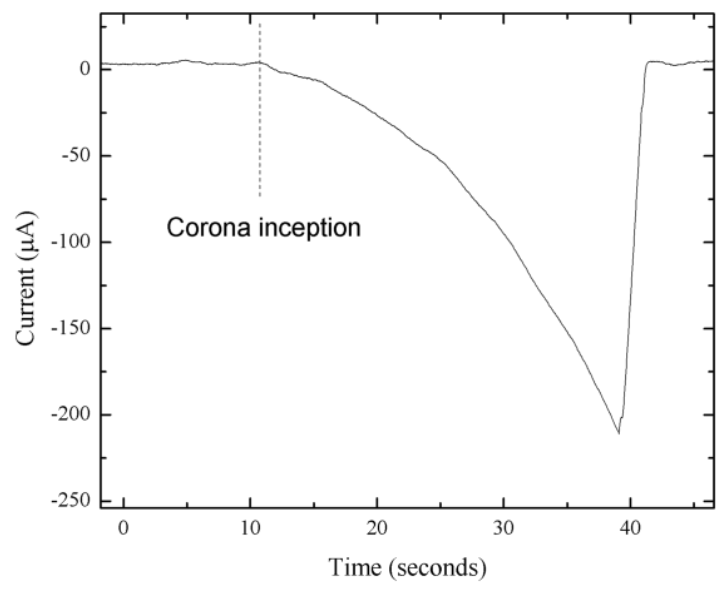

(b) Negative energisation

Figure 2. Pre-breakdown corona current in the switch: (a) positive energisation, (b) negative energisation. 
complete absence of this difference) in the positive and negative spark breakdown voltages, [2]. Therefore, it is expected that in the case of air-filled self-breakdown closing switch, a critical inter-electrode distance should exist: for inter-electrode gaps smaller than this critical distance, both positive and negative spark breakdown voltages will be almost the same, while for gaps larger than the critical distance, the negative spark breakdown voltage will be higher than the positive spark breakdown voltage. This behaviour can be used in practical plasma closing switches in order to provide operational performance independent of stress polarity.

In the present paper, the corona ignition voltage, spark breakdown voltage and pre-breakdown corona current have been measured in an air-filled plasma closing switch with a point-plane electrode configuration. Polarity effects on operational characteristics of this switch have been analysed.

\section{EXPERIMENTAL SET-UP}

In order to investigate operational characteristics of the switch (breakdown voltages and corona discharge parameters), a dedicated experimental set-up has been developed and constructed. The spark breakdown voltage, corona ignition voltage and corona current were obtained for the switch filled with bottled compressed air (room temperature $\sim 20^{\circ} \mathrm{C}, 12.5 \% \mathrm{rh}$ ) in the range of pressures from $0.1 \mathrm{MPa}$ to $0.35 \mathrm{MPa}$, for 8 inter-electrode gaps in the range between $0.5 \mathrm{~mm}$ and $14 \mathrm{~mm}$.

In these tests the point electrode was energised with steadily increasing voltage of both polarities until spark breakdown occurred. Figure 1 shows the experimental design where a Glassman HVDC power supply (EH series $0-60 \mathrm{kV}$ ) was used to provide up to $60 \mathrm{kV}$ to the point electrode via a $1 \mathrm{M} \Omega$ charging resistor. The voltage increase was controlled via a LabVIEW program interfacing with a National Instruments DAQ (USB6008) providing a ramped voltage with a stepped rate of $370 \mathrm{~V} / 150 \mathrm{~ms}$. When measuring pre-breakdown corona discharge current the switch was grounded via a $100 \mathrm{k} \Omega$ shunt resistor, the voltage drop across this shunt resistor was measured using a Tektronix P6015A HV probe (1000:1), and a Tektronix TDS 2024 (200 MHz, $2 \mathrm{GS} / \mathrm{s})$ oscilloscope, Figure 1. Corona inception voltage was determined as the voltage at which the corona current starts to show a clear tendency to increase (start to be above current noise fluctuations). Both positive and negative corona current waveforms were digitally filtered using adjacent-averaging procedure in Origin 8 software for clear identification of corona inception voltage.

The spark breakdown voltage was measured in a separate set of tests without the current viewing resistor, with the plane electrode of the switch directly connected to the ground. The breakdown voltage was identified at the moment of the collapse of voltage this voltage was

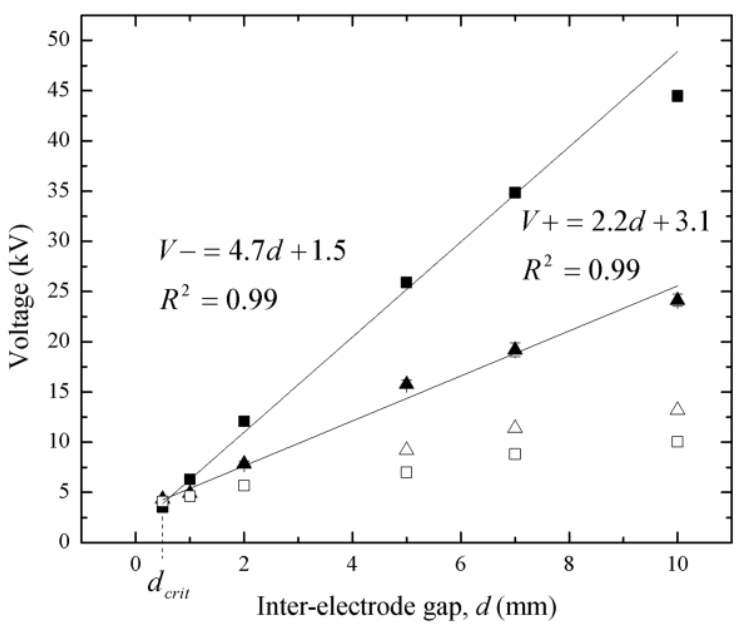

(a) Soft-tone gramophone needle, radius $0.036 \mathrm{~mm}$

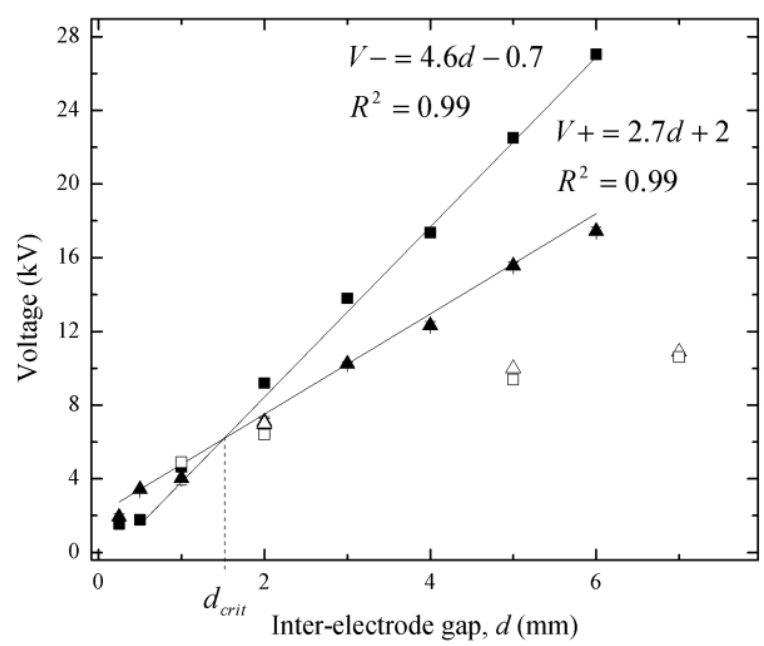

(b) Sharpened tungsten rod, radius $0.068 \mathrm{~mm}$.

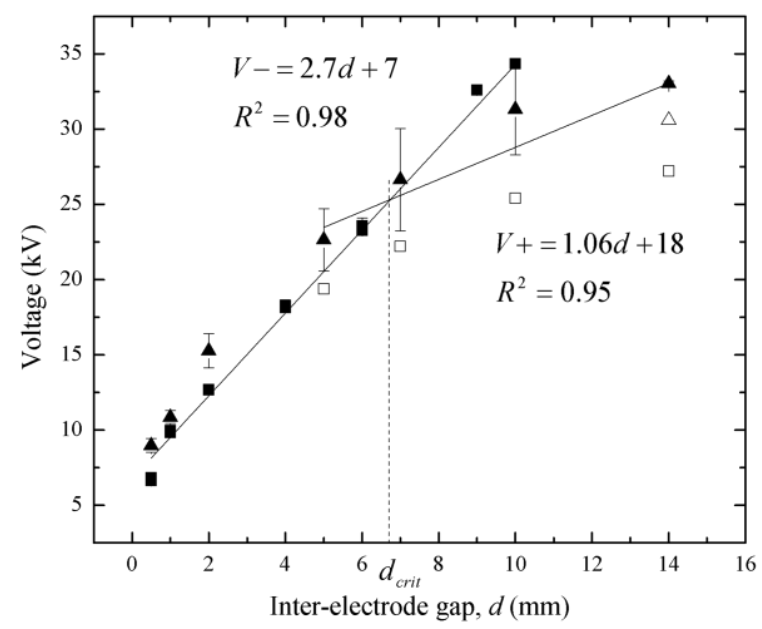

(c) Hemispherical tipped tungsten rod, radius $0.8 \mathrm{~mm}$.

Figure 3. Self-breakdown voltage as a function of inter-electrode gap for point-plane topology. Comparing positive and negative energisation breakdown in air at $0.3 \mathrm{MPa}$ for different radii of HV electrodes Squares - negative energisation; Triangles - positive energisation; Solid lines - best fitting line; Solid symbols - breakdown voltage; Open symbols - Corona inception voltages. Each point is an average value of 30 measurements, error bars represent standard deviation values. 
measured using a North Star PVM-5 HV probe (1000:1) and a Tektronix TDS 2024 (200 MHz, 2 GS/s) oscilloscope.

The localised field enhancement in the vicinity of the sharp high-tension electrode is strongly dependent on the radius of the point electrode, [15-18], and it is expected that the radius of the point electrode can affect the switching performance. Therefore, this paper investigates three different $\mathrm{HV}$ point electrodes: a tungsten rod with tip radius $0.068 \mathrm{~mm}$; a tungsten rod with tip radius $0.8 \mathrm{~mm}$; and a soft-tone gramophone needle with radius $0.036 \mathrm{~mm}$, since it has a well-defined shape and is often used in the power and pulsed power industries as a high-tension point electrode, [20]. The sharp point tungsten electrode with radius of $0.068 \mathrm{~mm}$ was used to create a strong field enhancement. In contrast, the hemispherical tipped rod with radius $0.8 \mathrm{~mm}$ creates $\sim 7 \mathrm{x}$ lower peak field, the peak field values in the point-plane topology have been calculated using the expression from [15]. Therefore, it is expected that for the larger radius electrode, corona current will be lower and space charge effects will be less pronounced. The ground plane electrode was a $50 \mathrm{~mm}$ diameter brass disk with rounded edges. A cylindrical switch body was made of black PVC.

The switch was set up with the desired point electrode and inter-electrode gap, and a rotary vacuum pump was used to evacuate the switch prior to filling with bottled air. The pressure in the switch was accurately set and controlled using a precision digital regulator Alicat PCD. The switch was evacuated every time when changing gas pressure, gap separation or electrode type.

Figure 2 shows typical pre-breakdown currents for positive and negative energisation of the point electrode, where the current waveform was obtained by division of the voltage measured across the shunt resistor by the nominal value of this resistor.

\section{RESULTS AND DISCUSSION}

Breakdown events were measured for all three point electrodes, with inter-electrode distances between $0.5 \mathrm{~mm}$ and $14 \mathrm{~mm}$, and for air pressures between $0.1 \mathrm{MPa}$ and $0.35 \mathrm{MPa}$. The point HV electrode was stressed with both positive and negative voltages and each breakdown measurement was repeated 30 times to allow statistical treatment of the results. Plotting the breakdown voltage as a function of inter-electrode gap shows the differences between breakdown voltages and corona characteristics for positive and negative energisation. These graphs have been plotted and analysed for all 7 pressures in $0.05 \mathrm{MPa}$ increments. Figure 3 shows an example of the analysis conducted in the present study: this figure shows positive and negative corona ignition and breakdown voltages at $0.3 \mathrm{MPa}$ for each of the point electrodes used. Images of the point $\mathrm{HV}$ electrodes were taken using an optical microscope with $1000 \mathrm{X}$ magnification: no visible change in point radius due to electrical erosion was visually observed after breakdown tests.

It was found that the positive corona ignition voltage was (slightly) higher than negative ignition voltage for all tested pressures, distances, and electrodes.

As can be seen in Figure 3, in the case of negative energisation, the spark breakdown voltage increases with an increase in the inter-electrode spacing. Experimental data on negative breakdown voltages as functions of the interelectrode gaps were fitted with straight lines over the whole range of the tested inter-electrode distances and for all pressures used in this study. These lines are shown on Figure 3, together with the analytical coefficients and goodness of fitting $\left(\mathrm{R}^{2}\right)$ values: $\mathrm{R}^{2}$ values of 0.98-0.99 are typical for all 3 points.

Positive breakdown voltages, $V_{b r}$, also increase with an increase in the inter-electrode spacing, however positive $V_{b r}$ demonstrates more complex behaviour as compared with negative $V_{b r}$. For sharper HV electrodes $(0.036 \mathrm{~mm}$ and $0.068 \mathrm{~mm}$ radii electrodes, Figures $3 \mathrm{a}$ and $3 \mathrm{~b}$ ) experimental data on positive breakdown voltage also have been fitted with straight lines in the full range of the tested distances and pressures. However, the gradient of these lines is lower than that for the lines plotted thought negative breakdown data. For example, in the case of $0.036 \mathrm{~mm}$ radius electrode (gramophone needle, Figure 3a, the gradient of the line of best fit is $2.24 \mathrm{kV} / \mathrm{mm}$ for positive breakdown voltages compared to $4.7 \mathrm{kV} / \mathrm{mm}$ for the negative breakdown data. The straight lines of best fit through the breakdown data for positive and negative energisation for $0.036 \mathrm{~mm}$ and $0.068 \mathrm{~mm}$ radius electrodes (Figures $3 \mathrm{a}$ and $3 \mathrm{~b}$ ) intersect indicating that there is a critical gap separation where the breakdown voltage is identical for both polarities of energisation. This gap length is referred to as $d_{c r i t}$ in this paper. The low values of $d_{\text {crit }}$ observed with tip radii of $0.036 \mathrm{~mm}$ and $0.068 \mathrm{~mm}$ (Figure $3 \mathrm{a}$ and $\mathrm{b}$ ) mean that there is insufficient data to draw conclusions as to whether the positive and negative breakdown data follow an identical trend over a range of gap lengths below $d_{\text {crit }}$.

For the electrode with the largest tip diameter, the tungsten rod with a $0.8 \mathrm{~mm}$ radius tip, the positive breakdown voltage can't be fitted with a single straight line over the whole range of tested gap spacing's. For shorter distances, the values of the breakdown voltage for both polarities of applied voltage are broadly similar and the fitting line for negative voltages can also be used to describe the functional behaviour of the positive breakdown voltages. For larger gap distances the positive breakdown voltages are lower than the negative voltages and positive breakdown data in this range from $5 \mathrm{~mm}$ to $14 \mathrm{~mm}$ were fitted with a second straight line, Figure 3c. This line intersects the line of the best fit for negative breakdown 
data and the intersection point defines $d_{\text {crit }}$ for this $0.8 \mathrm{~mm}$ radius electrode.

It was found that for the electrodes with smaller tip radii the standard deviation of positive breakdown voltage for all distances and pressures are significantly higher than those for negative energisation. This difference is even more marked in the case of the HV electrode with the largest tip radius $(0.8 \mathrm{~mm})$, where the standard deviation for positive breakdown voltage was an order of magnitude higher than for negative polarity, $\pm 5 \mathrm{kV}$ and $\pm 0.5 \mathrm{kV}$, respectively. Potentially this effect (higher deviation in positive spark breakdown voltages) can be a result of different mechanisms of initiation and development of positive and negative corona discharges in air: in the case of increasing of $\mathrm{HV}$ stress, the development of positive corona discharges progresses through different stages including a highly unstable and volatile impulsive (flashing) corona stage, while the behaviour of the negative corona discharges is more predictable, typically negative corona current is a combination of DC component and Trichel impulses, [21].

As discussed in Introduction, the critical gap, $d_{\text {crit }}$, in airfilled gap is defined by complex processes in the ionisation and transport zones in the inter-electrode gap including sheath formation and space charge screening. In the present section the existence of the critical gap has been confirmed experimentally. It was shown that for shorter gaps $\left(d<d_{c r i t}\right)$ there is no substantial difference in the positive and negative spark breakdown voltages (in such gaps positive and negative ions produce similar effect on the electric field). However, for larger gaps $\left(d>d_{\text {crit }}\right)$, the corona discharge under negative energisation injects an order of magnitude higher current as compared with positive energisation which results in more pronounced space charge effects and higher negative spark breakdown voltage.

The observed values of the critical gap, $d_{\text {crit }}$ depend on the pressure of the gas in the gap and the radius of the electrode tip. These critical distances have been plotted as functions of air pressure for all 3 electrodes, Figure 4 . It can be seen that as the pressure is increased, $d_{c r i t}$ becomes smaller. The critical gap for the $0.8 \mathrm{~mm}$ radius point electrode reduces from $\sim 10 \mathrm{~mm}$ at atmospheric pressure down to $\sim 8 \mathrm{~mm}$ at $0.15 \mathrm{MPa}$, a $20 \%$ reduction in critical gap with an increase of pressure of $0.05 \mathrm{MPa}$. However, with further increase in pressure up to $0.2 \mathrm{MPa}$, the reduction in $d_{\text {crit }}$ is smaller, $\sim 2.5 \%$. This trend continues and $d_{\text {crit }}$ appears to reach a constant value for higher pressures, $\sim 0.35 \mathrm{MPa}$. The critical gaps for the gramophone needle ( $0.036 \mathrm{~mm}$ radius) and tungsten point electrode $(0.068 \mathrm{~mm}$ radius) were similar to each other and significantly smaller than the critical gap for the $0.8 \mathrm{~mm}$ radius point electrode. However, the same trend in reduction of the critical gap for

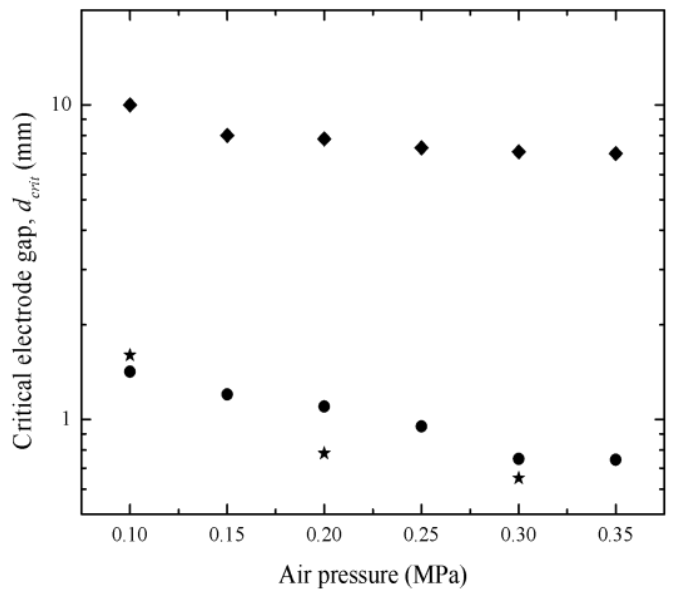

Figure 4. Critical inter-electrode gap at which positive energisation breakdown voltage reduced below negative energisation breakdown voltage as a function of pressure for 3 point electrodes in compressed air. Diamonds - hemispherical tipped tungsten rod, radius $0.8 \mathrm{~mm}$; Stars - soft tone gramophone needle, radius $0.036 \mathrm{~mm}$; Circles - sharpened tungsten rod, radius $0.068 \mathrm{~mm}$.

both of these sharp electrodes was observed: for gramophone needles, the gap reduces by $\sim 51 \%$, from $1.6 \mathrm{~mm}$ to $0.78 \mathrm{~mm}$, with an increase of air pressure from $0.1 \mathrm{MPa}$ to $0.2 \mathrm{MPa}$.

It was found that the critical distance reduces as the radius of the electrode increases, which can be explained by in the lower geometrical field enhancement which results in reduced corona induced space charge screening. Lower field enhancement at the tip of the point electrode means larger gaps are required to initiate corona discharge prior to breakdown. As the breakdown voltage in large gaps is strongly dependent on corona discharge, the critical gap is larger with larger radius. It is expected that electrode material will not have a significant impact on the critical distance because corona ignition voltage is not strongly dependent on electrode material, [2].

The appearance of detectable pre-breakdown corona current was used to record the corresponding corona ignition voltage. Negative corona current increases very gradually starting from a few $\mathrm{nA}$ (which is the detection limit in the present set-up) to a few $\mu \mathrm{A}$ as the voltage is increased. The negative corona discharge could start to impact the breakdown voltage from a low energisation level. In contrast, the positive corona current usually appears suddenly, transitioning from no detectable level to a few $\mu \mathrm{A},[5]$.

Examining the corona ignition voltage (indicated by open symbols in Figure 3) prior to complete spark breakdown shows that complete breakdown of the gap is not always preceded by intensive corona activity. The conditions where 
corona discharge occur prior to complete breakdown depend on applied voltage polarity, gas pressure and the radius of the point electrode, [22].

In these experiments, it was found that at shorter gaps (depending on pressure and electrode radius) there was no detectable pre-breakdown current that would be indicative of noticeable corona discharge for either positive or negative energisation. For example, in the case of the $0.036 \mathrm{~mm}$ radius $\mathrm{HV}$ electrode (Figure $3 \mathrm{a}$ ), there was no corona discharge at $0.5 \mathrm{~mm}$ inter-electrode spacing for pressures equal to and below $0.2 \mathrm{MPa}$.

In the cases where corona discharges appeared prior to spark breakdown, it was observed that negative energisation would produce detectable corona discharge at smaller gap spacing, whereas positive energisation leads directly to spark breakdown under similar conditions. Figure 3 a shows corona ignition voltages (open symbols) for negative energisation starting from $1 \mathrm{~mm}$, however positive corona was not detected appearing until an inter-electrode spacing of $2 \mathrm{~mm}$.

The probability of corona discharge appearing before complete breakdown was dependent on the air pressure in the switch. As pressure is increased, the probability that corona will precede spark breakdown increases for the sharp point electrodes. For example, at $0.1 \mathrm{MPa}$, using a $0.036 \mathrm{~mm}$ radius $\mathrm{HV}$ electrode, no corona was detected for $0.5 \mathrm{~mm}, 1 \mathrm{~mm}$ or $2 \mathrm{~mm}$ electrode gaps. At $0.35 \mathrm{MPa}$, negative corona discharges precede complete breakdown in all inter-electrode gaps; while corona activity starts at $2 \mathrm{~mm}$ for positive energisation.

The appearance of corona discharge before breakdown is also highly dependent on the radius of the point electrode. The sharper electrodes produced corona discharges prior to breakdown under both polarities of energisation for similar gap distances $(1-4 \mathrm{~mm})$. In the case of the $0.8 \mathrm{~mm}$ radius electrode, corona discharge before breakdown was observed for smaller gap distances $(\sim 5 \mathrm{~mm})$ under negative energisation as compared with positive energisation $(\sim 10$ $\mathrm{mm})$. This can clearly be seen in Figure $3 \mathrm{c}$ where negative corona discharge is observed before breakdown for gaps greater than $5 \mathrm{~mm}$ while positive corona discharge is only observed for a gap of $14 \mathrm{~mm}$.

Comparison of positive and negative breakdown voltages shows the effect of such corona activity: due to the development of volumetric space charge the breakdown voltage is higher for negative energisation as compared with positive energisation in gaps greater than $d_{c r i t}$, and this difference increases with an increase in the inter-electrode spacing, Figure 3. For distances shorter than $d_{\text {crit }}$, the difference in the behaviour of the positive and negative breakdown voltages is not well pronounced; potentially this

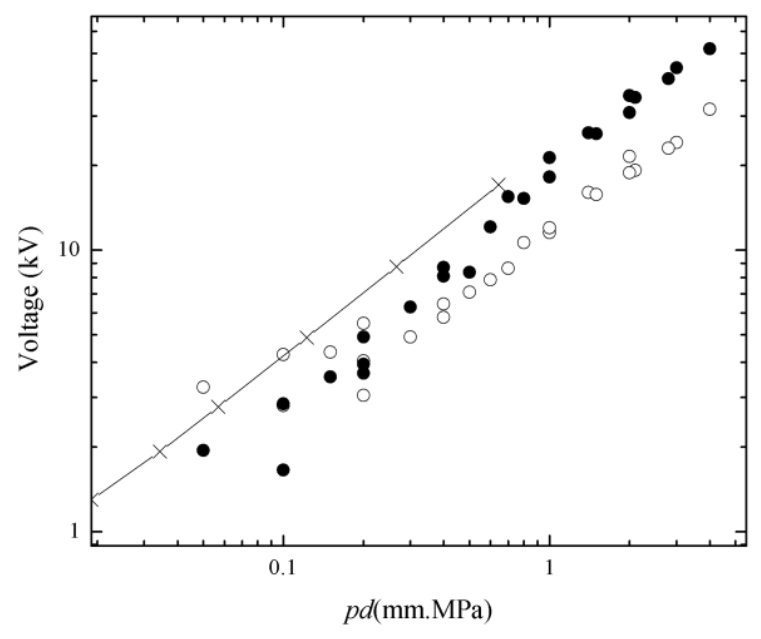

(a) Gramophone needle, radius $0.036 \mathrm{~mm}$.

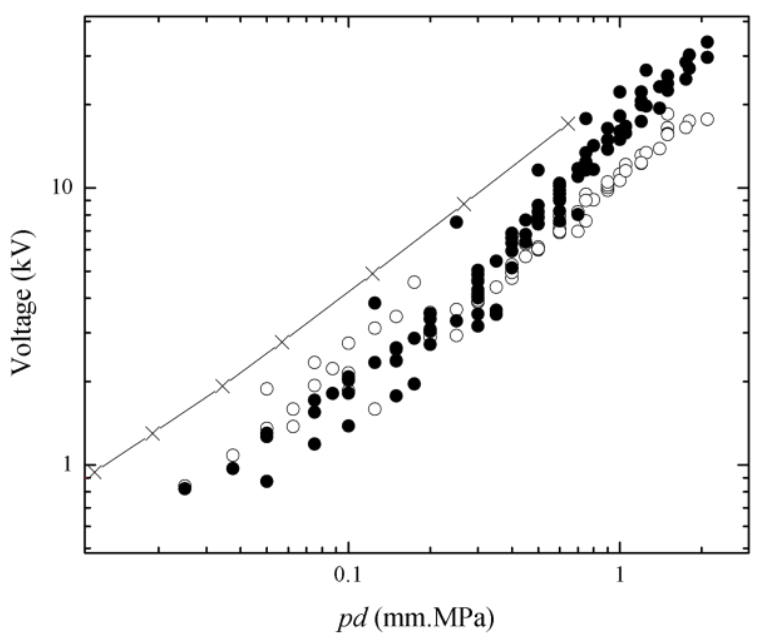

(b) Sharpened tungsten rod, radius $0.068 \mathrm{~mm}$.

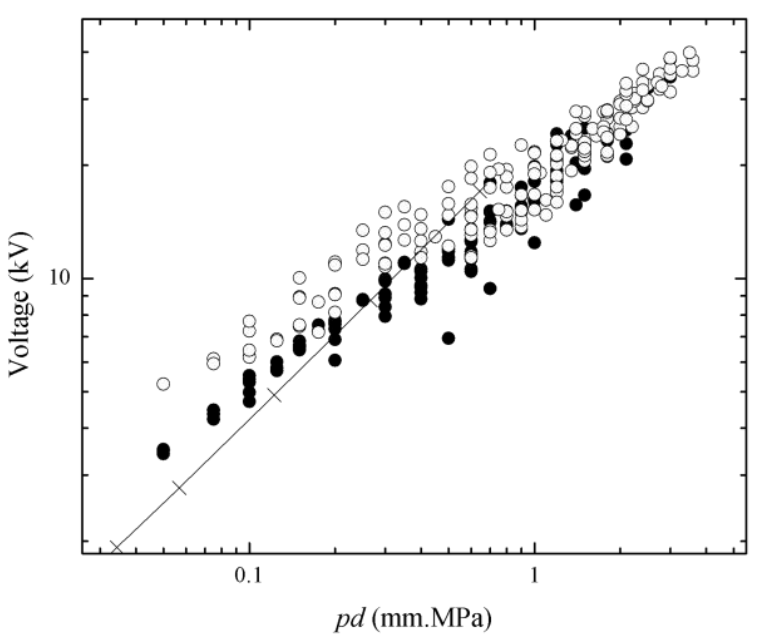

(c) Hemispherical tipped tungsten rod, radius $0.8 \mathrm{~mm}$.

Figure 5. Self-breakdown voltage as a function of pressure and interelectrode gap, $p d$ for 3 different point electrodes along with a classic Paschen curve from literature [25]. Crosses with line - Paschen curve [25]; Black markers - negative energisation; White markers - positive energisation. 
is a result of presence of the positive and negative charges which in short gaps produce comparable space charge effects. With an increase in the gap, the effects produced by the volumetric negative space charge continue to govern the negative breakdown voltage behaviour (almost linear increase in $V_{b r}$ with an increase in the gap spacing). However, in the case of positive energisation the field screening effect produced by a weaker positive space charge is not so effective as the positive corona current for this range of distances and electrode radii is significantly lower that the negative current at the same level of the applied voltage. Thus, the rate of rise of the positive breakdown voltage with an inter-electrode distance decreases. In the case of $0.8 \mathrm{~mm}$ gap, no positive corona current was observed for gaps shorter than $14 \mathrm{~mm}$ which results in deviation of the positive breakdown voltages from the negative ones at longer inter-electrode gaps (larger critical distance, Figure 3c).

It is common to display breakdown voltages of gaseous dielectrics as a function of the product of gas pressure and inter-electrode separation, $p d,[1,2,10,23,24]$. The breakdown voltage of uniform topologies can be predicted given the inter-electrode gap and gas pressure using Paschen's law, [22, 23].

The breakdown voltages in non-uniform topologies obtained in the present study also have been plotted as $V_{b r}(p d)$ functions for all pressures and electrode radii. Figure 5 shows the obtained breakdown voltages of each of the three electrodes as a function of the product of pressure and distance $(p d)$ alongside the right branch of conventional Paschen breakdown curve (at values of $p d$ above the Paschen minimum). Conventional Paschen data for air were taken from the literature, [25] in order to compare the results obtained in the present paper with already published $V_{b r}(p d)$ data. Figure 5 shows that, the data obtained in the present work closely follows the conventional Paschen's law, and there is a strong scaling tendency for breakdown voltages in highly divergent electric fields which can be utilised in the design of plasma closing switches and other component parts of compact high-voltage systems.

\section{CONCLUSION}

In this paper, an air-filled plasma closing switch with a highly non-uniform and variable point-plane topology has been developed in order to investigate its performance under positive and negative ramp voltage stress. Corona ignition and spark breakdown voltage have been obtained for pressures in the range of $0.1-0.35 \mathrm{MPa}$, and the switch was energised using a computer-controlled high-voltage system until breakdown occurred. Three different point electrodes were used: tungsten rods with radii $0.068 \mathrm{~mm}$ and $0.8 \mathrm{~mm}$, and a gramophone needle with radius $0.036 \mathrm{~mm}$.
It was observed that negative breakdown voltage increases linearly with increasing inter-electrode gap throughout the range of gaps tested. At very short gaps (depending on radius of point electrode), positive breakdown voltage is comparable to negative breakdown voltage. For larger gaps, with an increase in intensity of pre-breakdown negative corona discharges, and as a result more pronounced negative space charge, the positive breakdown voltage becomes lower than the negative breakdown voltage, and the rate of its increase with the distance reduces. The critical distance, $d_{\text {crit }}$, at which positive and negative breakdown voltages deviate from each other has been identified for all tested pressures and electrode topologies. The negative pre-breakdown corona discharges initiate at much smaller gaps than positive corona discharges, causing stabilisation in the switch operation at much smaller gaps, [2].

It was found that the critical distance is dependent on the $\mathrm{HV}$ point electrode radius and the gas pressure. With the larger radius needle, $0.8 \mathrm{~mm}$, the critical distance at atmospheric pressure $(0.1 \mathrm{MPa})$ is $\sim 5$-fold larger than the critical distance for the sharper electrodes. It was found that as the gas pressure is increased the critical distance reduces. This is likely due to the increased pre-breakdown corona activity at shorter gaps that is present at higher pressures. The critical distance and corresponding pressure can be used for identification of operational regimes in which corona stabilisation is necessary or vice versa - regimes when it is desirable to achieve the identical operational characteristics of the switch for both polarities, positive and negative. The results obtained and discussed in this paper aid in the design, development and optimisation of the performance of HV systems whether breakdown is desired or not: for example, plasma closing switches require breakdown at predictable levels, whereas designers of compact high-voltage/pulsed power systems require experimental data to ensure the avoidance of unwanted breakdown.

\section{ACKNOWLEDGMENT}

This research has been part-funded by a UK MoD research programme.

\section{REFERENCES}

[1] E. Kuffel, W. S. Zaengl, and J. Kuffel, High Voltage Engineering, Fundamentals, Newnes Oxford, 2000.

[2] J. M. Meek, and J. D. Craggs, Electrical Breakdown of Gases, John Wiley, New York, 1953.

[3] A. Maglaras, and F. V. Topalis, "Influence of ground and corona currents on dielectric behavior of small air gaps", IEEE Trans. Dielectr. Electr. Insul., Vol. 16, No. 1, pp. 32-41, 2009.

[4] A. L. Maglaras, K. Giannakopoulou, T. G. Kousiouris, F. V. Topalis, D. S. Katsaros, and L. A. Maglaras, "Control and optimization of the Corona effects and breakdown of small rod-plate air gaps stressed by dc and impulse voltages", IEEE Int'l. Conf. Solid Dielectr. (ICSD), pp. 160-165, 2013.

[5] A. F. Kip, "Positive-Point-to-Plane Discharge in Air at Atmospheric Pressure", Phys. Rev., Vol. 54, No. 2, pp.139-146, 1938. 
[6] M. Xiaobo, C. Changlong, and L. Wang, "Corona Charge Injection Prior to Sparkover in an Inversed Rod-Plane Gap Under Composite Voltages", IEEE Trans. Power Delivery, Vol. 27, No. 3, pp. 1442$1449,2012$.

[7] L. K. Warne, R. E. Jorgenson, and E. E. Kunhardt, "Criterion for spark-breakdown in non-uniform fields", J. Appl. Phys., Vol. 115, No. 14, pp. 143303, 2014.

[8] J. R. Beveridge, S. J. MacGregor, M. J. Given, and I. V. Timoshkin, "A Corona-stabilised Plasma, Closing Switch," IEEE Trans. Dielectr. Electr. Insul., Vol. 16, No. 4, pp. 948-955, 2009.

[9] M. G. Hogg, I. V. Timoshkin, S. J. Macgregor, M. P. Wilson, M. J. Given, and T. Wang, "Electrical breakdown of short non-uniform air gaps", IEEE 19th Pulsed Power Conference (PPC), pp. 1-4, 2013.

[10] D. S. Antao, D. A. Staack, A. Fridman, and B. Farouk, "Atmospheric Pressure DC Corona Discharges: Operating Regimes and Potential Applications", Plasma Sources Sci. Technol., Vol. 18, No. 3, pp. 35016, 2009.

[11] P. Chowdhuri, A. K. Mishra and B. W. McConnell, "Volt-time characteristics of short air gaps under nonstandard lightning voltage waves", IEEE Trans. on Power Delivery, Vol. 12, No. 1, pp. 470476, 1997.

[12] Y. Akishev, M. Grushin, I, Kochetov, V. Karal'nik, A. Napartovich and N. Trushkin, "Negative corona, glow and spark discharges in ambient air and transitions between them", Plasma Sources Sci. Technol., Vol. 14, No. 2, pp. 18-25, 2005.

[13] Th. Hinterholzer and W. Boeck, "Breakdown in $\mathrm{SF}_{6}$ influenced by Corona-stabilisation", Int'l. Conf. Electr. Insul. Dielectr. Phenomena, pp. 413-416, 2000.

[14] R. Sigmong, "Simple approximate treatment of unipolar spacecharge-dominated coronas: The Warburg law and the saturation current”, J. Appl. Phys.,Vol. 53, No. 2, pp. 891-898, 1982.

[15] R. Coelho, and J. Debeau, "Properties of the tip-plane configuration", J. Phys. D: Appl. Phys., Vol. 4, pp. 1266-1280, 1970.

[16] K. Adamiak, V. Atrazhev, and P. Atten, "Corona discharge in the hyperbolic point-plane configuration: direct ionization criterion versus an approximate formulations", IEEE Trans. Dielectr. Electr. Insul., Vol. 12, No. 5, pp. 1015-1024, 2005.

[17] I. V. Timoshkin, S. J. MacGregor, M. J. Given, J. R. Beveridge, and J. M. Lehr, "Dynamic Characteristics Of Corona Discharges In Point-Plane Electrode Topologies Influenced By Space Charge", IEEE Int'l. Power Modul. High Voltage Conf., pp. 495-498, 2008.

[18] M. Hogg, I. Timoshkin, S. MacGregor, M. Given, M. Wilson, and T. Wang, "Performance of Air-Filled Plasma Closing Switch with Corona Pre-ionisation", XIX Int'l. Conf. Gas Discharges and Their Applications, pp. 504-507, 2012.

[19] H. Bluhm, Pulsed Power Systems: Principles and Applications Springer, Berlin, 2006.

[20] IEEE Standard Techniques for High-Voltage Testing (Revision of IEEE Std 4-1 978). IEEE Std 4- 1995: pp. i-129.

[21] I. V. Timoshkin, M. Maclean, M. P. Wilson, M. J. Given, S. J. MacGregor, T. Wang and J. G Anderson, "Bactericidal Effect of Corona Discharges in Atmospheric Air", IEEE Trans. Plasma Sci., Vol. 40, No. 10, pp. 2322-2333, 2012.

[22] F.W. Peek, Dielectric Phenomena in High Voltage Engineering, McGraw-Hill Book Company, London, 1915.

[23] F. Paschen, "Ueber die zum Funkenübergang in Luft, Wasserstoff und Kohlensäure bei verschiedenen Drucken erforderliche Potentialdifferenz," Annalen der Physik, Vol. 273, No. 5, pp. $69-$ 96, 1889.

[24] L.B. Loeb, Fundamental Processes of Electrical Discharge in Gases, John Wiley and Sons, New York, 1939.

[25] S.C. Brown, Basic Data of Plasma Physics, 1966.

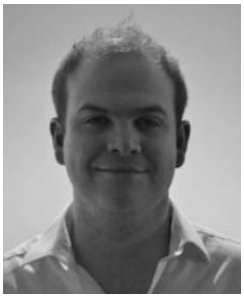

Michael G Hogg is a Ph.D. degree student in the Department of Electronic and Electrical Engineering, University of Strathclyde, Glasgow. He received his B.Eng. (Hons) degree in Electronic and Electrical Engineering from the University of Strathclyde in 2010 and his M.Res. in 2011. His research interests include electrical breakdown in environmentally friendly gases, plasma closing switches, corona discharge and water as a dielectric insulator.

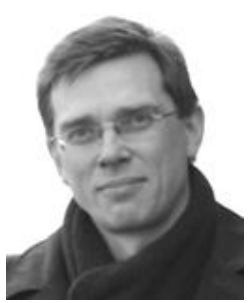

Igor V. Timoshkin (M'07-SM'14) received the degree in physics from the Moscow State University (Russia) in 1992, and the Diploma and the Ph.D. degree from the Imperial College of Science, Technology and Medicine, (London, UK) in 2001. After graduation from MSU he worked as a Researcher at Moscow State AgroEngineering University, and then at the Institute for High Temperatures of Russian Academy of Sciences before moving to ICSTM in 1997. He joined the Department of Electronic and Electrical Engineering of the University of Strathclyde (Glasgow, UK) in 2001 as an Academic Visitor, where he became a Senior Lecturer in 2011. His research interests include properties of solid and liquid dielectric materials, electronics of plasma discharges in condensed media, practical applications of electro-hydraulic and high-power ultrasound pulses, bio-dielectrics and effects of electromagnetic fields on biological objects.

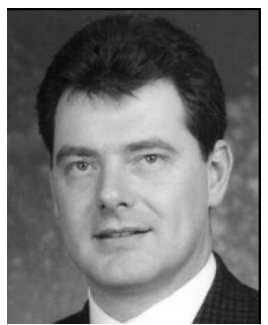

Scott J. MacGregor (M'95-SM'14) received the B.Sc. and Ph.D. degrees from the University of Strathclyde, Glasgow, U.K., in 1982 and 1986, respectively. He became a Pulsed Power Research Fellow in 1986 and a Lecturer in pulsed-power technology in 1989. In 1994, he became a Senior Lecturer, with a promotion to Reader and Professor of High Voltage Engineering, in 1999 and 2001, respectively. From 2010 he became the Dean of the Engineering Faculty of the University of Strathclyde. His research interests include high-voltage pulse generation, high-frequency diagnostics, high-power repetitive switching, high-speed switching, electronic methods for food pasteurization and sterilization, generation of high-power ultrasound (HPU), plasma channel drilling, pulsed-plasma cleaning of pipes, and stimulation of oil wells with HPU.

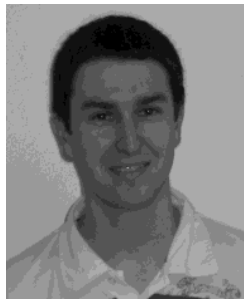

Mark P. Wilson (M'10) was born in Stranraer, Scotland, in 1982. He received the B.Eng. (with honours), M.Phil., and Ph.D. degrees in electronic and electrical engineering from the University of Strathclyde, Glasgow, U.K., in 2004, 2007, and 2011, respectively. He is presently working as a Teaching Associate at the University of Strathclyde, where he continues to investigate surface flashover of solids immersed in insulating oil. Mark is a member of the IEEE Nuclear and Plasma Sciences Society, from whom he received a Graduate Scholarship Award in 2011, the IEEE Dielectrics and Electrical Insulation Society, and the IET. 


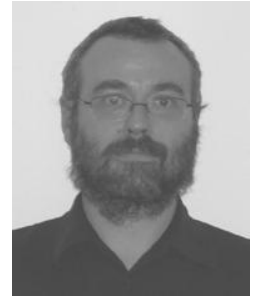

Martin J. Given (M'99-SM'11) is currently a Senior Lecturer in the Department of Electronic and Electrical Engineering at the University of Strathclyde. He received a degree in physics from the University of Sussex in 1981 and a $\mathrm{PhD}$ in electronic and electrical engineering from the University of Strathclyde in 1996. His research interests include, ageing processes and condition monitoring in solid and liquid insulation systems, high speed switching and pulse power applications. 